\title{
Probióticos en diarrea aguda infecciosa
}

\author{
MARIANA KAHN CH. ${ }^{1}$, FRANCISCA FUENTES D. ${ }^{2}$, GUISELLA VILLARROEL M. ${ }^{2}$ \\ 1. Servicio de Salud Metropolitano Norte. \\ 2. Pontificia Universidad Católica de Chile.
}

\begin{abstract}
\section{Probiotics in acute infectious diarrhea}

Background: Acute infectious diarrhea in children represents a worldwide problem and it relates to desnutrition, morbility and death. Objective: Determine the usefulness of probiotics in the treatment of acute infectious diarrhea in children by reviewing the scientific information available in the form of metaanalysis. Methods: A detailed search was performed in electronic databases. Four of the articles found met inclusion criteria. Results: The four meta-analysis studied demonstrated that the administration of probiotics in children with acute infectious diarrhea decreased the episode duration in approximately one day with minimal adverse events. Conclusions: Despite methodological limitations of the analysed articles, the studies showed the same trend towards observing a benefit in the use of probiotics in acute infectious diarrhea in children. The latter supports the consistency of this association. In general, the use of probiotics in children with acute infectious diarrhea would be useful in general practice reducing diarrhea duration in approximately one day.

(Key words: acute diarrhea, probiotics, children).

Rev Chil Pediatr 2009; 80 (2): 129-136
\end{abstract}

\section{RESUMEN}

Antecedentes: La diarrea aguda infecciosa en niños constituye un problema a nivel mundial y se traduce en desnutrición, morbilidad y muerte. Objetivos: Determinar la utilidad de los probióticos en el tratamiento de la diarrea aguda infecciosa en los niños mediante la revisión de la información científica disponible en forma de meta-análisis. Material y Métodos: Se realizó una búsqueda detallada en bases de datos electrónicas de uso frecuente. Cuatro de los artículos encontrados cumplían con los criterios de inclusión. Resultados: Los cuatro meta-análisis revisados evidenciaron que la administración de probióticos en niños con diarrea aguda infecciosa disminuía la duración del cuadro en aproximadamente un día con mínimos efectos adversos. Conclusiones: A pesar de las limitaciones metodológicas de los trabajos analizados, la mayoría de los estudios presentan una misma tendencia al observar beneficio en el uso de los probióticos en diarrea aguda infecciosa en niños. Lo anterior le otorga mayor consistencia a la asociación. En general, el uso de probióticos en niños con diarrea aguda infecciosa sería útil en la práctica clínica diaria, disminuyendo la duración de la diarrea en aproximadamente un día.

(Palabras clave: diarrea aguda, probióticos, niños).

Rev Chil Pediatr 2009; 80 (2): 129-136

Trabajo recibido el 20 de octubre de 2008, devuelto para corregir el 18 de noviembre de 2008, segunda versión el 19 de enero de 2009, tercera versión el 17 de febrero de 2009, aceptado para publicación el 23 de febrero de 2009.

Correspondencia a:

Dra. Mariana Kahn Ch.

E-mail: marianakahnchernilo@hotmail.com 


\section{Introducción}

La diarrea se define como un cambio en el tránsito intestinal de un individuo en particular, caracterizada por un aumento en el contenido líquido, volumen y, usualmente, de la frecuencia de las deposiciones ${ }^{1,2}$. La terapia más importante para la diarrea aguda asociada a deshidratación es la rehidratación oral. Sin embargo, ésta no produce una disminución sustancial de la duración del episodio diarreico ni tampoco una disminución del volumen de las deposiciones. Lo anterior hace importante el estudio de terapias complementarias ${ }^{3}$.

La diarrea infecciosa ocurre más frecuentemente en países en vías de desarrollo que en aquéllos industrializados ${ }^{4}$. En los países subdesarrollados los niños experimentan en promedio seis a 12 episodios de diarrea aguda al año en comparación a dos episodios en Estados Uni$\operatorname{dos}^{5}$. La diarrea aguda usualmente es autolimitada en los países desarrollados; sin embargo, en aquéllos en desarrollo este tipo de enfermedad puede traducirse en desnutrición, morbilidad asociada y muerte ${ }^{6}$. En los países en desarrollo las muertes son más frecuentes en los niños menores de cinco años, y dan cuenta de 2,4 a 3,3 millones de muertes cada año ${ }^{7}$.

A nivel mundial, el rotavirus es el agente más frecuente productor de diarrea aguda severa y de mortalidad asociada a diarrea en niños ${ }^{8}$. Dentro de los agentes bacterianos destacan: Escherichia coli enterotoxigénica, Salmonella, Shighella, Yersinia, Campylobacter, y Vibrio cholera. Los parásitos más frecuentes que producen diarrea son Cryptosporidium y Giardia ${ }^{9}$. La etiología infecciosa de esta enfermedad dependerá de factores locales como la disponibilidad de agua potable y medidas sanitarias ${ }^{10}$.

El tratamiento actual de la diarrea aguda es principalmente de soporte e incluye primariamente manejo sintomático. Recientemente, los probióticos han sido propuestos como terapia adyuvante en el tratamiento en la diarrea aguda en los niños. Los probióticos son un grupo de organismos bacterianos y fúngicos que se cree tendrían efectos beneficiosos en la salud humana cuando colonizan el intestino. Los probióticos, que incluyen a los Lactobacillus y bífidobac- terias, podrían inhibir a los patógenos entéricos mediante la secreción de agentes antibacterianos ${ }^{11}$. Adicionalmente, la competencia por receptores de la mucosa intestinal podría prevenir la adhesión y sobrecrecimiento de bacterias gram negativas aerobias enterotóxicas ${ }^{12} y$ virus enteropatogénicos ${ }^{13,14} \mathrm{y}$ de esta manera permitir la adherencia de organismos más beneficiosos a la superficie intestinal. El aumento de la producción de ácidos grasos volátiles y la reducción del $\mathrm{pH}$ fecal podrían también jugar un rol crucial en la inhibición de los organismos enterotóxicos ${ }^{15}$. Además, la ingestión de Lactobacillus $G G$ podría ejercer efectos inmunomoduladores que disminuirían la inflamación intestinal $^{16,17}$.

Los probióticos más conocidos son las bacterias ácido lácticas y el hongo Saccharomy$\operatorname{ces}^{18}$. Cepas probióticas similares pueden tener efectos clínicos distintos, por lo que el comité experto de la Organización de las Naciones Unidas para la Agricultura y la Alimentación (FAO) y la Organización Mundial de la Salud (OMS) enfatizan que los resultados beneficiosos observados en una cepa no pueden extrapolarse a otras cepas similares ${ }^{19}$.

La mayoría de los estudios sobre los probióticos apoyan su eficacia en el tratamiento de la diarrea aguda. A pesar de esto, no son utilizados ampliamente por los pediatras.

El presente trabajo pretende analizar críticamente la evidencia existente hasta este momento con respecto al uso de probióticos en el manejo de la diarrea aguda infecciosa en los niños.

\section{Material y Métodos}

\section{Objetivos}

El objetivo de la presente revisión es determinar la efectividad clínica de los probióticos en el tratamiento de la diarrea aguda infecciosa en los niños.

\section{Pregunta Clínica}

¿Son efectivos clínicamente los probióticos en el tratamiento de la diarrea aguda infecciosa en niños?

1. Pacientes: Niños con diarrea aguda infecciosa. 
2. Intervención: Probióticos.

3. Control: Placebo o no intervención.

4. Evento de interés:

- Primario: Características clínicas de la diarrea (duración, frecuencia y volumen de deposiciones).

- Secundario: Efectos adversos.

5. Tipo de estudio: Ensayos clínicos controlados randomizados, revisiones sistemáticas y meta-análisis.

\section{Métodos y Estrategias de Búsqueda}

Búsqueda bibliográfica: Se realizó primero una búsqueda bibliográfica en bases de datos sin filtro de evidencia: Medline, Lilacs y SciELO. La búsqueda en Medline se realizó mediante las palabras clave "Diarrhea" [MeSH] OR "Antidiarrheals" [MeSH] AND "Therapeutics" [MeSH] OR "Therapy" [MeSH] AND "Probiotics" [MeSH] en artículos publicados los últimos diez años hasta Agosto de 2008. La búsqueda en Lilacs y SciELO se realizó mediante las palabras clave "diarrea", "antidiarreicos" y "probióticos" sin límite de fecha de publicación. Luego se realizó una búsqueda biliográfica con filtro de evidencia en la Biblioteca Cochrane con la palabra clave "probiótico". Los criterios de inclusión empleados fueron metodológicos y temáticos. Con respecto a los criterios metodológicos se privilegió en primer lugar los estudios analíticos en seres humanos que aportaran evidencia directa sobre tratamiento, es decir, ensayos clínicos controlados randomizados, revisiones sistemáticas y metaanálisis. Con respecto a los criterios temáticos se consideraron artículos con resumen, en inglés, español o francés cuyos sujetos tuviesen hasta 18 años de edad.

\section{Resultados}

La búsqueda inicial en Medline según los criterios previamente descritos dio como resultado 64 artículos, debido a lo cual se restringió aún más la búsqueda sólo a meta-análisis, obteniendo siete estudios. De éstos, tres fueron eliminados por referirse al uso de probióticos en diarrea asociada a antibióticos y otro fue eliminado porque analizaba sólo la vía de administración. A través del ítem "artículos relacionados" se encontró un cuarto meta-análisis. En la Biblioteca Cochrane se encontró un artículo (Biblioteca Cochrane Plus, Base de Datos Cochrane de Revisiones Sistemáticas, número 3,2008 ) que se eliminó debido a que no cumplía con el objetivo de la presente revisión como es determinar la efectividad clínica de los probióticos en el tratamiento de la diarrea aguda infecciosa en los niños, ya que incluía a sujetos de más de 18 años de edad y no presentaba análisis por subgrupo de edad con respecto a duración, frecuencia y volumen de las deposiciones, ni tampoco de efectos adversos. En los buscadores SciELO y Lilacs no se encontraron artículos. Finalmente, se analizaron cuatro metaanálisis.

\section{Huang JS, Bousvaros A, Lee J, Díaz A, Davidson E. Efficacy of probiotic use in acute diarrhea in children: a meta-analysis. Dig Dis Sci 2002; 47(11): 2625-34.}

En el trabajo de Huang et $\mathrm{al}^{20}$, el objetivo fue determinar la eficacia de los probióticos mediante la reducción de la duración de los días de diarrea aguda en niños sanos menores de cinco años. Los criterios de inclusión fueron: estudios randomizados con grupos control claro, con comparación directa entre grupos de tratamiento y control, y que la duración de la diarrea estuviese reportada como evento de interés. Se encontraron 18 artículos.

Componentes de la pregunta clínica: $\mathrm{Pa}-$ cientes: niños sanos menores de cinco años con diarrea aguda infecciosa. Intervención: uso de probióticos. Control: placebo o no intervención. Evento de interés: duración del episodio diarreico en días.

Resultados: Similares entre los estudios. Se observó una disminución del número de días de diarrea en 0,8 días (IC 95\% 0,6-1,1; $\mathrm{p}<0,001$ ). Se encontró considerable heterogeneidad metodológica entre estudios (cuando se habla de heterogeneidad se pueden distinguir dos aspectos: por un lado la heterogeneidad metodológica con respecto a las diferencias existentes entre los estudios en cuanto a características de los pacientes incluidos, la metodología utilizada, el tiempo de seguimiento, las dosis empleadas, la localización geográfica, etc, y por otro lado, el concepto de heterogeneidad estadística, que 
únicamente cuantifica la variabilidad entre los resultados de los estudios, y que puede ser debida a las diferencias reales de planteamiento y ejecución entre los estudios incluidos, o a otras causas). Para disminuir la heterogeneidad metodológica se realizaron sub-análisis de: Estudios en hospitalizados, doble ciego, de Lactobacillus y otros probióticos. No fueron evaluados efectos adversos, tampoco volumen ni frecuencia de las deposiciones.

Comentarios: La edad de los pacientes fue entre uno y 60 meses. La dosis aportada fue de diez millones hasta diez billones de unidades formadoras de colonias/día en sales de rehidratación oral estándar, no reportada en todos los estudios. Días de tratamiento: dos a cinco, en 13 de los estudios (en el resto no reportado o variable). Catorce de los estudios correspondieron a pacientes hospitalizados, dos incluyeron hospitalizados y ambulatorios. No hubo sesgo de publicación. Aunque los resultados de este trabajo fueron considerablemente heterogéneos desde el punto de vista metodológico, la restricción del análisis sólo a estudios doble ciego realizados en pacientes hospitalizados y que evaluaran Lactobacillus (particularmente GG), redujo significativamente la heterogeneidad metodológica medida.

\section{Szajewska H, Skórka A, Dylag M. Meta-} analysis: Saccharomyces boulardii for treating acute diarrhea in children. Aliment Pharmacol Ther 2007; 25: 257-64.

En el trabajo de Szajewska et al ${ }^{3}$, el objetivo fue determinar la efectividad de Saccharomyces boulardii en el tratamiento de la diarrea aguda infecciosa en los niños. Se incluyó estudios randomizados controlados que comparasen $S$. boulardii con placebo o no intervención adicional. El evento de interés primario fue la duración de la diarrea, volumen de deposiciones, porcentaje de niños con diarrea en variados intervalos de tiempo (especificados por investigadores) y el porcentaje de niños con diarrea de más de siete días. El evento de interés secundario se refirió a la frecuencia de deposiciones, vómitos, adherencia (aceptación del tratamiento) y efectos adversos. Se identificaron cinco artículos, de los cuales tres fueron con placebo y dos sin intervención.
Componentes de la pregunta clínica: $\mathrm{Pa}-$ cientes: niños menores de 12 años con diarrea aguda infecciosa. Intervención: uso de Saccharomyces boulardii. Control: placebo o no intervención adicional. Evento de interés: duración del episodio diarreico en días, frecuencia de las deposiciones y efectos adversos.

Resultados: Similares entre los estudios. Se observó una disminución del número de días de diarrea en 1,1 días (IC 95\% 0,83-1,3). Esto fue analizado en cuatro de los cinco estudios, observándose homogeneidad estadística, para este evento de interés. Se observó una reducción de la frecuencia de deposiciones para aquéllos tratados con $S$. boulardii en comparación con control (excepto el día uno). No se reportaron efectos adversos en ninguno de los estudios. No se mencionó volumen de las deposiciones. Los estudios incluidos no fueron considerablemente heterogéneos desde el punto de vista metodológico.

Comentarios: La edad de los participantes varió entre dos meses y 12 años, la dosis diaria fue de $250-750 \mathrm{mg}$. La duración de la intervención fue de cinco a seis días. Hubo heterogeneidad metodológica con respecto al origen de los pacientes (tres estudios en hospitalizados, un estudio en ambulatorio y uno que no especifica). La calidad metodológica varió entre los estudios. Aunque los estudios incluidos no fueron significativamente heterogéneos desde el punto de vista metodológico, las conclusiones estadísticas con respecto a los determinantes de este tipo de heterogeneidad pudieron estar erradas debido al escaso número de estudios. Aún más, no se pudo excluir completamente el sesgo de publicación. Aunque se realizó análisis estadístico con respecto al sesgo de publicación, los autores concluyeron que este test tenía poco poder en un meta-análisis de este tipo debido al escaso número de estudios. Sin embargo, el trabajo tendría mayor peso ya que sólo analizó un tipo de microorganismo probiótico.

Szajewska H, Skórka A, Ruszczynski D. Meta-analysis: Lactobacillus GG for treating acute diarrhea in children. Aliment Pharmacol Ther 2007; 25: 871-81.

En otro trabajo de Szajewska et $\mathrm{al}^{21}$, el objetivo fue determinar la efectividad de Lacto- 
bacillus $G G$ en el tratamiento de la diarrea aguda infecciosa en los niños. Se incluyó estudios randomizados controlados que comparasen Lactobacillus $G G$ con placebo o no intervención adicional. El evento de interés primario fue la duración de la diarrea y volumen de deposiciones. El evento de interés secundario se refirió a porcentaje de niños con diarrea en variados intervalos de tiempo (especificados por investigadores), el porcentaje de niños con diarrea de más de siete días, la frecuencia de deposiciones, vómitos y efectos adversos. Se identificaron ocho artículos, de los cuales cuatro fueron con placebo, dos con sales de rehidratación oral y dos sin intervención.

Componentes de la pregunta clínica: $\mathrm{Pa}-$ cientes: niños menores de cinco años con diarrea aguda infecciosa. Intervención: uso de Lactobacillus GG. Control: placebo, sales de rehidratación oral o no intervención adicional. Evento de interés: duración del episodio diarreico en días, volumen de las deposiciones y efectos adversos.

Resultados: Similares entre los estudios. Se observó una disminución del número de días de diarrea en 1,1 días (IC 95\% 0,3-1,9). Esto fue analizado en siete estudios. Sin embargo, la significancia se perdió en el análisis de sensibilidad excepto para los estudios con ciego. En el análisis por subgrupo basado en el país de origen se evidenció que este hallazgo era significativo sólo para los estudios europeos. Dos estudios presentaron datos sobre volumen de deposiciones; no existió diferencia significativa entre ambos grupos. Se reportaron efectos adversos sólo en un estudio (mioclonías en dos pacientes, uno del grupo control y otro del tratamiento). No se mencionó frecuencia de las deposiciones. Se encontró heterogeneidad estadística significativa en: volumen total de las deposiciones, duración de la diarrea, duración de la diarrea por rotavirus, de la diarrea de causa desconocida y de la duración de la estadía hospitalaria. Sin embargo, este tipo de heterogeneidad no fue significativa para otros eventos de interés.

Comentarios: La edad de los participantes varió entre uno y 36 meses. La dosis diaria del producto en estudio, la presentación (leche o cápsulas) y la duración de la intervención varia- ron entre los estudios. Hubo heterogeneidad metodológica con respecto al origen de los pacientes (cinco estudios en hospitalizados, un estudio en ambulatorio y dos mixtos). Otra limitación del trabajo incluyó tamaño de muestra pequeña en los distintos estudios. Los artículos incluidos eran significativamente heterogéneos desde el punto de vista metodológico, esto se debió probablemente a las diferencias entre los pacientes, origen y dosis de probiótico.

\section{Van Niel C, Feudtner C, Garrison M, Dimitri $A$. Lactobacillus therapy for acute infectious diarrhea in children: a meta-analysis. Pediatrics 2002; 109: 678-84.}

En el trabajo de Van Niel et $\mathrm{al}^{22}$, el objetivo fue determinar si el tratamiento con Lactobacillus mejoraba el resultado clínico de los niños con diarrea aguda infecciosa. Se incluyó estudios adecuadamente randomizados controlados y ciegos en los cuales el grupo tratamiento recibiera Lactobacillus y el grupo control un placebo adecuado, y que existiese reportes clínicos de la intensidad de la diarrea. El evento de interés primario fue la intensidad de la diarrea (duración, frecuencia y volumen de deposiciones). El evento de interés secundario se refirió a necesidad de intervención médica adicional (como hidratación intravenosa) y reacciones adversas como resultado del tratamiento. Se identificaron nueve artículos.

Componentes de la pregunta clínica: $\mathrm{Pa}-$ cientes: niños menores de 5 años con diarrea aguda infecciosa. Intervención: Lactobacillus. Control: placebo. Evento de interés: duración del episodio diarreico en días, frecuencia de las deposiciones y efectos adversas.

Resultados: Similares entre los estudios. Se observó una disminución del número de días de diarrea (análisis de siete estudios) en 0,7 días (IC 95\% 0,3-1,2). Además, se encontró una disminución en la frecuencia de deposiciones (análisis de tres estudios) en el segundo día de diarrea de 1,6/día (IC 95\% 0,7-2,6). No se pudo examinar frecuencia de deposiciones en otros días ni volumen de éstas. No se encontró diferencias al sub-analizar por país desarrollado, sólo Lactobacillus vivo o diarrea por todas las causas (no sólo rotavirus). En todos los estudios incluidos las reacciones adversas que fue- 
ran consistentes con signos y síntomas usualmente asociados a diarrea infecciosa ocurrieron por igual en ambos grupos, excepto en dos estudios que reportaron disminución de los vómitos en el grupo de Lactobacillus. Un estudio reportó efectos adversos fuera del espectro clínico: mioclonías fueron observadas en un paciente del grupo tratamiento y en uno del control. El análisis de los pacientes que requirieron intervención adicional no fue posible debido a que los datos eran insuficientes. Existió una relación dosis-respuesta en los ocho estudios que reportaron duración de la diarrea. Se observó una asociación lineal positiva significativa entre el log de la dosis de Lactobacillus y la reducción de la duración de la diarrea en días $(\mathrm{p}<0,01)$. Esto se observaría con la administración de más de diez billones de unidades formadoras de colonias en las primeras 48 horas de inicio de la diarrea.

Comentarios: La edad de los participantes varió entre uno y más de 36 meses. La dosis diaria del producto en estudio, la presentación (leche o cápsulas) y la duración de la intervención variaron. Hubo heterogeneidad metodológica con respecto al origen de los pacientes: ocho estudios en hospitalizados y un estudio mixto en donde la mayoría de los pacientes eran hospitalizados. A pesar de que la mayoría de los pacientes eran hospitalizados casi todos requirieron sólo hidratación oral y además algunos estudios excluyeron a los pacientes con deshidratación severa, por lo que los resultados podrían extrapolarse a la población ambulatoria con diarrea.

En la tabla 1 se observa un cuadro comparativo resumen de los cuatro meta-análisis previamente revisados.

\section{Discusión}

Se deben considerar las limitaciones metodológicas de algunos estudios: no hay definición clara de la enfermedad (del inicio ni del término del episodio diarreico agudo), inicio del tratamiento, randomización inadecuada o poco clara, ausencia de ciego, falta de cálculo de tamaño de muestra (tanto para efectividad clínica como para seguridad), sin análisis de intención a tratar, seguimiento impreciso de los pacientes, muestras pequeñas y de origen heterogéneo, distintos tipos y cepas de probióticos en diferentes dosis y presentaciones, variados eventos de interés y análisis de etiología no siempre presente.

Los probióticos analizados en los cuatro metaanálisis revisados son heterogéneos (probióticos en general, Saccharomyces boulardii, Lactobacillus y Lactobacillus $G G$ ) al igual que el origen y edad de los pacientes, así como la etiología de la diarrea aguda. De los 40 estudios incluidos en los meta-análisis, 30 de éstos incluyen pacientes únicamente hospitalizados, cinco estudios comprenden pacientes hospitalizados y ambulatorios, dos estudios incluyen sólo pacientes ambulatorios y en tres trabajos no se especifica el origen de los pacientes. Lo anterior contrasta con la realidad epidemiológica local donde la incidencia de diarrea aguda es baja y se concentra en los dos primeros años de vida, la mayoría de los episodios diarreicos agudos son tratados en forma ambulatoria, con mínimo riesgo de evolución a diarrea persistente y con etiología preferente por rotavirus y otros virus.

No existen estudios en Chile que analicen la efectividad clínica de los probióticos en la diarrea aguda en los niños, por lo que los resultados observados en los trabajos y la posible aplicabilidad de este tipo de terapia en nuestro país deben ser tomados con precaución, debido a las diferencias entre nuestra población de niños con diarrea aguda y la de los pacientes incluidos en los meta-análisis.

Otro punto a considerar es el costo de este tipo de terapias en nuestro país, que en promedio fluctúa entre los cinco y seis mil pesos ( 8 a 10 dólares). Considerando que el riesgo de diarrea aguda en los niños aumenta con las malas condiciones higiénicas y el hacinamiento, la mayoría de estos cuadros se concentran en población de escasos recursos que no tiene los medios económicos para adquirir este tipo de tratamiento.

Uno de los trabajos analizados refiere la aparición de mioclonías en un paciente del grupo tratamiento y en un paciente del grupo control, pero no queda claro si ese efecto adverso 
Tabla 1. Cuadro comparativo resumen de los cuatro meta-análisis revisados

\begin{tabular}{|c|c|c|c|c|}
\hline & $\begin{array}{l}\text { Huang et al. } \\
\qquad(2002)^{20}\end{array}$ & $\begin{array}{l}\text { Szajewska et al. } \\
(2007)^{3}\end{array}$ & $\begin{array}{l}\text { Szajewska et al. } \\
\qquad(2007)^{21}\end{array}$ & $\begin{array}{l}\text { Van Niel et al. } \\
(2002)^{22}\end{array}$ \\
\hline Revista & $\begin{array}{l}\text { Digestive Diseases } \\
\text { Sciences }\end{array}$ & $\begin{array}{l}\text { Alimentary Pharmacology } \\
\& \text { Therapeutics }\end{array}$ & $\begin{array}{l}\text { Alimentary Pharmacology } \\
\& \text { Therapeutics }\end{array}$ & Pediatrics \\
\hline $\begin{array}{l}\text { Tipo de estudios } \\
\text { incluidos }\end{array}$ & $\begin{array}{l}\text { Ensayos clínicos } \\
\text { randomizados }\end{array}$ & $\begin{array}{l}\text { Ensayos clínicos } \\
\text { randomizados }\end{array}$ & $\begin{array}{l}\text { Ensayos clínicos } \\
\text { randomizados }\end{array}$ & $\begin{array}{l}\text { Ensayos clínicos } \\
\text { randomizados }\end{array}$ \\
\hline Pacientes & $\begin{array}{l}\text { Niños menores de } \\
\text { cinco años }\end{array}$ & $\begin{array}{l}\text { Niños menores de } \\
12 \text { años }\end{array}$ & $\begin{array}{l}\text { Niños menores de } \\
\text { tres años }\end{array}$ & $\begin{array}{l}\text { Niños menores de } \\
\text { cinco años }\end{array}$ \\
\hline $\begin{array}{l}\text { Origen de los } \\
\text { pacientes }\end{array}$ & $\begin{array}{l}14 \text { estudios en hospi- } \\
\text { talizados, dos estudios } \\
\text { en pacientes hospitali- } \\
\text { zados y ambulatorios, } \\
\text { dos estudios no se } \\
\text { especifica origen }\end{array}$ & $\begin{array}{l}\text { Tres estudios en hospi- } \\
\text { talizados, uno en pa- } \\
\text { cientes ambulatorios } \\
\text { y uno no se especifica } \\
\text { origen }\end{array}$ & $\begin{array}{l}\text { Cinco estudios en hos- } \\
\text { pitalizados, uno en } \\
\text { pacientes ambulatorios } \\
\text { y dos estudios en pa- } \\
\text { cientes hospitalizados } \\
\text { y ambulatorios }\end{array}$ & $\begin{array}{l}\text { Ocho estudios en hospi- } \\
\text { talizados y un estudio en } \\
\text { pacientes hospitalizados } \\
\text { y ambulatorios }\end{array}$ \\
\hline $\begin{array}{l}\text { Número de } \\
\text { estudios incluidos }\end{array}$ & 18 & 5 & 8 & 9 \\
\hline $\begin{array}{l}\text { Variable } \\
\text { independiente }\end{array}$ & Probiótico & Saccharomyces boulardii & Lactobacillus GG & Lactobacillus \\
\hline $\begin{array}{l}\text { Variable } \\
\text { dependiente }\end{array}$ & $\begin{array}{l}\text { Duración del episodio } \\
\text { diarreico en días }\end{array}$ & $\begin{array}{l}\text { Duración del episodio } \\
\text { diarreico en días, fre- } \\
\text { cuencia de las deposi- } \\
\text { ciones y efectos adversos }\end{array}$ & $\begin{array}{l}\text { Duración del episodio } \\
\text { diarreico en días, volu- } \\
\text { men de las deposicio- } \\
\text { nes y efectos adversos }\end{array}$ & $\begin{array}{l}\text { Duración del episodio } \\
\text { diarreico en días, fre- } \\
\text { cuencia de las deposi- } \\
\text { ciones y efectos adversos }\end{array}$ \\
\hline $\begin{array}{l}\text { Estimador } \\
\text { calculado }\end{array}$ & $\begin{array}{l}\text { Se observó una dismi- } \\
\text { nución del número de } \\
\text { días de diarrea en } 0,8 \\
\text { días }\end{array}$ & $\begin{array}{l}\text { Se observó una dismi- } \\
\text { nución del número de } \\
\text { días de diarrea en } 1,1 \\
\text { días y una reducción } \\
\text { de la frecuencia de las } \\
\text { deposiciones (excepto } \\
\text { en el día uno) sin apa- } \\
\text { rición de efectos } \\
\text { adversos }\end{array}$ & $\begin{array}{l}\text { Se observó una dismi- } \\
\text { nución del número de } \\
\text { días de diarrea en } 1,1 \\
\text { días, sin diferencia en } \\
\text { el volumen de las de- } \\
\text { posiciones. Mioclonías } \\
\text { en un paciente del } \\
\text { grupo tratamiento y } \\
\text { en otro del grupo } \\
\text { control }\end{array}$ & $\begin{array}{l}\text { Se observó una dismi- } \\
\text { nución del número de } \\
\text { días de diarrea en } 0,7 \\
\text { días; además, se encon- } \\
\text { tró una disminución en } \\
\text { la frecuencia de deposi- } \\
\text { ciones en el segundo día } \\
\text { de diarrea de } 1,6 / \text { día. } \\
\text { Mioclonías en un pacien- } \\
\text { te del grupo tratamiento } \\
\text { y en otro del grupo con- } \\
\text { trol. Menos vómitos en } \\
\text { el grupo tratamiento }\end{array}$ \\
\hline $\begin{array}{l}\text { Intervalo de } \\
\text { confianza }\end{array}$ & $\begin{array}{l}\text { IC } 95 \% \text { 0,6-1,1 } \\
\text { (días de diarrea) }\end{array}$ & $\begin{array}{l}\text { IC } 95 \% \text { 0,8-1,3 } \\
\text { (días de diarrea) }\end{array}$ & $\begin{array}{l}\text { IC } 95 \% \text { 0,3-1,9 } \\
\text { (días de diarrea) }\end{array}$ & $\begin{array}{l}\text { IC } 95 \% 0,3-1,2 \\
\text { (días de diarrea) } \\
\text { IC } 95 \% 0,7-2,6 \\
\text { (frecuencia de deposicio- } \\
\text { nes) }\end{array}$ \\
\hline
\end{tabular}

estaría o no relacionado con la administración del probiótico en estudio. Otro trabajo evidenció menos vómitos en el grupo tratamiento. Sin embargo, cabe destacar que no existe análisis con respecto al cálculo de tamaño de muestra ni para determinar efectividad clínica ni seguridad del tratamiento.

Llama la atención que el evento de interés más importante en los cuatro meta-análisis fue la duración en días de la diarrea, a pesar de que la OMS recomienda como criterio cuantitativo para evaluar agentes terapéuticos el volumen de las deposiciones ${ }^{23}$.

Dado que los episodios de diarrea aguda en su mayoría no presentan complicaciones, son autolimitados y no requieren un tratamiento específico, es necesario determinar mediante el análisis de la relación costo-efectividad si los probióticos deben usarse en grupos de pacientes determinados. Por ejemplo, la aparente efi- 
cacia de los probióticos en la reducción de la duración de la diarrea aguda puede ser especialmente importante en los países en vías de desarrollo, donde la diarrea aguda infantil es un factor de riesgo de la diarrea persistente (más de 14 días) que, a su vez, está estrechamente vinculada a la desnutrición ${ }^{10}$.

A pesar de las limitaciones previamente descritas, la mayoría de los estudios presentan una misma tendencia al observar beneficio en el uso de los probióticos en diarrea aguda infecciosa en niños con escasos efectos adversos.

En general, el uso de probióticos en niños con diarrea aguda infecciosa podría ser útil en la práctica clínica diaria. Sin embargo, la aplicabilidad de este tipo de tratamientos en una comunidad como la nacional con baja incidencia de diarrea aguda, menor intensidad de los episodios y mínimo riesgo de evolucionar a diarrea persistente no es tan evidente como en otros lugares en donde los episodios diarreicos agudos sí tienen riesgo de traducirse en morbilidad asociada, desnutrición y muerte.

\section{Referencias}

1.- WHO: The Treatment of Diarrhea. A Manual for Physicians and other Senior Health Workers; WHO/ CDR/95.3. Geneva: World Health Organization, 1995.

2.- Riedel BD, Ghishan FK: Acute diarrhea. In: Walker WA, Durie PR, Hamilton HR, Walker-Smith JA, Watkins JB, eds. Pediatric Gastrointestinal Disease, $2^{\text {nd }}$ edn. St Louis: Mosby-Year Book Inc., 1996: 25162.

3.- Szajewska H, Skórka A, Dylag M: Meta-analysis: Saccharomyces boulardii for treating acute diarrhea in children. Aliment Pharmacol Ther 2007; 25: 257 64.

4.- Guerrant RL, Hughes JM, Crane J: Diarrhea in developed and developing countries: magnitude, special settings, an etiologies. Rev Infect Dis 1990; 12 (1): 41-50.

5.- Savarino SJ, Bourgeois AL: EpiDiarrheal disease: current concepts and future challenges. Epidemiology of diarrheal diseases in developed countries. Trans Roy Soc Trop Med Hyg 1993; 87 (3): 7-11.

6.- The magnitude of the global problem of diarrheal disease: a ten-year update. Bulletin of the World Health Organization 1992; 70 (6): 705-14.

7.- Anonymous: Persistent diarrhea in children in developing countries: memorandum from a WHO meeting. Bulletin of the World Health Organization 1988; 66 (6): 709-17.

8.- Cunliffe NA, Kilgore PE, Bresee JS, et al: Epidemiology of rotavirus diarrhea in Africa: a review to assess the need for rotavirus immunization. Bulletin of the World Health Organization 1998; 76 (5): 525-37.

9.- Huilan $S$, Zhen LG, Mathan MM, et al: Etiology of acute diarrhea among children in developing countries: a multicentre study in five countries. Bulletin of the World Health Organization 1991; 69 (5): 549-55.

10.- Walker-Smith JA: Diarrheal disease: current concepts and future challenges. Malnutrition and infection. Trans Roy Soc Trop Med Hyg 1993; 87 (3): 13-5.

11.- Jack RW. Tagg JR, Ray B: Bacteriocins of grampositive bacteria. Microbiol Rev 1995; 59: 171-200.

12.- Neeser JR, Granato D, Rouvet M: Lactobacillus johnsonnii La1 shares carbohydrate-binding specificities with several enteropathogenic bacteria. Glycobiology 2000; 10 (11): 1193-9.

13.- Duffy LC, Zielezny MA, Riepenhoff-Talty M: Reduction of virus shedding by B. bifidum in experimentally induced MRV infection. Dig Dis Sci 1994; 39: 233440.

14.-Duffy LC, Zielezny MA, Riepenhoff-Talty M: Effectiveness of Bifidobacterium bifidum in mediating the clinical course of murine rotavirus diarrhea. Pediatr Res 1994; 35(6): 690-5.

15.- Ohya T, Marubashi T, Ito $H$ : Significance of fecal volatile fatty acids in shedding of Escherichia coli 0157 from calves: experimental infection and preliminary use of a probiotic product. J Vet Med Sci 2000; 62 (11): 1151-5.

16.- Pelto L, Isolauri E, Lilius EM, Nuutila J, Salminem S: Probiotic bacteria down-regulate the milk-induced inflammatory response in milk-hypersensitive subjects but have an immunostimulatory effect in healthy subjects. Clin Exp Allergy 1998; 28: 1479-9.

17.- Majamaa H, Isolauri E: Probiotics: a novel approach in the management of food allergy. J Allergy Clin Immunol 1997; 99: 179-85.

18.- Naidu AS, Bidlack WR, Clemens RA: Probiotic spectra of lactic acid bacteria (LAB). Crit Rev Food Sci Nutr 1999; 39 (1): 13-126.

19.- Food and Agriculture Organization of the United Natios (FAO)/World Health Organization (WHO): Joint FAO/WHO expert consultation on evaluation of health and nutritional properties of probiotics in food including powder milk and live lactic bacteria. http:// ftp.fao.es/esn/food/probio_report_en.pdf 2001.

20.- Huang JS, Bousvaros A, Lee J, Díaz A, Davidson E: Efficacy of probiotic use in acute diarrhea in children: a meta-analysis. Dig Dis Sci 2002; 47 (11): 2625-34.

21.- Szajewska H, Skórka A, Ruszczynski D: Meta-analysis: Lactobacillus $G G$ for treating acute diarrhea in children. Aliment Pharmacol Ther 2007; 25: 87181.

22.- Van Niel C, Feudtner C, Garrison M, Dimitri A: Lactobacillus therapy for acute infectious diarrhea in children: a meta-analysis. Pediatrics 2002; 109: 67884.

23.- World Health Organization: The rational use of drugs in the management of acute diarrhea in children. Geneva, Switzerland: World Health Organization, 1990. 